\title{
Accuracy Limits in the Computed Transients on Overhead Lines Due to Inaccurate Ground Return Modeling
}

\author{
Adam Semlyen, Life Fellow, IEEE
}

\begin{abstract}
Electromagnetic transients on overhead transmission lines are strongly affected by the resistivity $\rho$ of ground return which, however, is often poorly known. Therefore, the calculation of transients with very high accuracy for a given $\rho$ may not make good engineering sense if it entails loss of computational efficiency. The effect of $\rho$ on the transients comes primarily via the ground return impedance. In order to judge what accuracy is reasonable, this paper derives the sensitivities of the ground return impedances for line and ground modes with respect to the resistivity $\rho$. The results are simple and may assist to decide on the accuracy of computations in EMTP-type simulation programs.
\end{abstract}

Index Terms-Electromagnetic transients, frequency dependence, ground return, sensitivity analysis, transmission lines.

\section{INTRODUCTION}

$\mathbf{F}$ OR almost three decades, the ecomputation of electromagnetic transients on overhead lines has been performed at different levels of accuracy. Thus, the first simulations using recursive convolutions [1] used only two exponentials for the fitting of the step response related to the propagation function and a single exponential for the characteristic admittance; but soon, very high accuracy was achieved by frequency domain fitting of order higher than ten [2]. The accuracy is even higher in some programs, such as in [3], where vector fitting [4] is used. However, it was always widely known that the inaccuracy of the ground return modeling - due mainly to the poor knowledge of the ground resistivity $\rho$ and also to the assumption of uniform and homogeneous ground-could strongly affect the accuracy of the results to the point that the high accuracy of the simulations is not warranted. In [5], this concern has resulted in less accurate but simpler and faster computations, particularly for the ground mode where the problem is more pronounced.

The purpose of this paper is to quantify the effect of uncertainty in our knowledge of $\rho$ on the accuracy of the computation of transients. This is possible due to the simple analytical relation between $\rho$ and the line inductances $L$, based on the concept of complex depth [6]; it permits the closed-form calculation of sensitivities. Most transients are affected directly by the ground return impedance, i.e., by $L$ (which now is complex). For instance, the voltage increase in open circuit due to charging currents (Ferranti effect) is due to the voltage rise on

Manuscript received November 9, 1999. This work was supported by the Natural Sciences and Engineering Research Council of Canada.

The author is with the Department of Electrical and Computer Engineering, University of Toronto, Toronto, ON M5S 3G4 Canada (e-mail: adam.semlyen@utoronto.ca).

Publisher Item Identifier S 0885-8977(02)05911-3. the line impedance; and, of course, voltage drops determine the short-circuit currents. These phenomena are usually influenced by ground return effects and only exceptionally will they be of pure line mode. Actually, on a transmission line with horizontal configuration, we have the strongest ground return effect on the ground mode, the $\beta$-mode is less affected and the weakest influence is on the $\alpha$-mode. Nonetheless, we examine both line and ground modes to clarify the validity of the expectation that the latter is the most important.

Using the "right" accuracy for fitting each transfer function in the transient simulation program permits a speed-up that is particularly important for simplified versions used for real-time simulations [7], for instance, for protective relaying. It is also important when building external system equivalents [8], which are usually formulated separately for the line and the ground mode, to choose the accuracy proper for each mode.

In the following we examine the dependence on the ground resistivity of inductances and thereby of the related resistances $R$ and reactances $X$. Sensitivities are partial derivatives, i.e., local functions. However, since the phenomena examined extend over a wide range of values for $\rho$ and of frequencies $f$, the variations of $R$ and $X$ and of the related sensitivities are shown in function of $\rho / f$, on which they depend directly.

\section{ANALYTICAL DERIVATIONS}

\section{A. Expressions for $L, R$, and $X$}

For the calculation of sensitivities, i.e., partial derivatives of the form $S=\partial L / \partial \rho$, we consider a horizontal conductor configuration because then, as we shall show in Section II-D, there are physical reasons for anticipating low sensitivities for the two line modes $\alpha$ and $\beta$. The three conductors, namely, $a, b$, and $c$, of radius $r$, are at height $h$ and the horizontal separation is $H$. The effect of the ground return is taken into account by the complex depth [6]

$$
p=\sqrt{\frac{\rho}{j \omega \mu_{0}}}
$$

which represents the depth below the surface of the ground of a reflective plane giving the position of the conductor images. We denote by $D$ the (complex) distance of a conductor to its image

$$
D=2(h+p) \text {. }
$$

In the expressions we derive below, $D$ should be viewed as the main (or independent variable) because it is a direct function 
of $\rho$ via $p$ of (1a). Thus, according to [6], the expressions of the (complex) self and mutual inductances of the inductance matrix

$$
L=\left[\begin{array}{ccc}
s & m & n \\
m & s & m \\
n & m & s
\end{array}\right]
$$

are

$$
\begin{aligned}
& s=L_{a a}=L_{b b}=L_{c c}=k \ln \frac{D}{r} \\
& m=L_{a b}=L_{b c}=k \ln \frac{\sqrt{D^{2}+H^{2}}}{H} \\
& n=L_{a c}=k \ln \frac{\sqrt{D^{2}+(2 H)^{2}}}{2 H}
\end{aligned}
$$

where $k=\mu_{0} /(2 \pi)$.

The geometry of the line suggests the use of $\alpha, \beta$, and zero components for both voltages and currents

$$
v_{\alpha \beta 0}=T v, i_{\alpha \beta 0}=T i, L_{\alpha \beta 0}=T^{-1} L T=T^{T} L T
$$

with the (ortho-normal) transformation matrix

$$
T=\left[\begin{array}{ccc}
\frac{1}{\sqrt{6}} & \frac{1}{\sqrt{2}} & \frac{1}{\sqrt{3}} \\
-\frac{2}{\sqrt{6}} & 0 & \frac{1}{\sqrt{3}} \\
\frac{1}{\sqrt{6}} & -\frac{1}{\sqrt{2}} & \frac{1}{\sqrt{3}}
\end{array}\right] .
$$

The columns of (5), corresponding to the $\alpha, \beta$, and 0 components, are close but not all identical to the eigenvectors of $L$ (only the second column, the $\beta$-mode, is an exact eigenvector) and therefore the similarity transformation of $L$ in (3) will yield $L_{\alpha \beta 0}$ with only a partial decoupling (namely, for the $\beta$-mode)

$$
L_{\alpha \beta 0}=\left[\begin{array}{ccc}
L_{\alpha} & 0 & L_{\alpha 0} \\
0 & L_{\beta} & 0 \\
L_{0 \alpha} & 0 & L_{0}
\end{array}\right]
$$

where

$$
\begin{aligned}
L_{\alpha} & =s-\frac{4}{3} m+\frac{1}{3} n \\
L_{\beta} & =s-n \\
L_{0} & =s+\frac{4}{3} m+\frac{2}{3} n \\
L_{\alpha 0} & =L_{0 \alpha}=\frac{\sqrt{2}}{3}(n-m) .
\end{aligned}
$$

Numerical evaluation of the inductances (7) shows that, for any reasonable set of parameters, the coupling inductance $L_{\alpha 0}$ is by approximately one order of magnitude smaller than the main inductances. It is therefore justified to view the $\alpha, \beta$, and 0 components as quasi-modes.

We now substitute the expressions for $s, m$, and $n$ from (3) into (7), condense the arguments under a single logarithm and then, taking into account that in general $H \ll|D|$, we do judiciously truncated expansions in terms of $H / D$. Thus, we obtain

$$
\begin{aligned}
L_{\alpha} & =k \ln \frac{H}{2^{1 / 3 r}}-k \frac{H^{4}}{D^{4}} \\
L_{\beta} & =k \ln \frac{2 H}{r}-2 k \frac{H^{2}}{D^{2}} \\
L_{0} & =3 k \ln \frac{D}{\sqrt[3]{4^{1 / 3} r H^{2}}}+2 k \frac{H^{2}}{D^{2}} \\
L_{\alpha 0} & =L_{0 \alpha}=\sqrt{2} k \ln \frac{1}{2^{1 / 3}}+\frac{k}{\sqrt{2}} \frac{H^{2}}{D^{2}} .
\end{aligned}
$$

These approximations of (7) and (3) are highly accurate. For $H /|D|$ ranging from 0.1 to 0.001 , the relative error of the main inductances does not exceed $10^{-4}$ and $10^{-12}$, respectively. Actually, the error in $L_{\alpha}$ is by about two orders of magnitude smaller because of the higher order approximation. On the other hand, the relative error in the coupling inductance $L_{\alpha 0}$ is by one order of magnitude larger because $L_{\alpha 0}$ is itself relatively small.

We note that, with the exception of $L_{0}$, the dominant logarithmic terms in the inductances (8a) do not depend on $D$ and thereby on $\rho$. Therefore, when taking derivatives, we expect to obtain very simple expressions for the sensitivities [see (9)]. It is already apparent from (8a) by inspection that the highest sensitivity will be for $L_{0}$, the lowest for $L_{\alpha}$.

For the calculation of relative sensitivities of $L_{i}$ from (8a), the absolute sensitivities $S_{i}=\partial L_{i} / \partial \rho(i=\alpha, \beta, 0)$ have to be related to some base inductance values. Since the ideal, lossless, inductances combine with the line capacitances to produce the travel delay, we shall subtract from (8a) these ideal values. The latter correspond to $\omega=\infty$ or $p=0$ and are obtained from (8a) by setting $D=2 H$. The result of the subtraction is

$$
\begin{aligned}
& L_{\alpha_{\Delta}}=-k\left(\frac{H^{4}}{D^{4}}-\frac{H^{4}}{(2 h)^{4}}\right) \\
& L_{\beta_{\Delta}}=-2 k\left(\frac{H^{2}}{D^{2}}-\frac{H^{2}}{(2 h)^{2}}\right) \\
& L_{0_{\Delta}}=3 k \ln \frac{D}{2 h}+2 k\left(\frac{H^{2}}{D^{2}}-\frac{H^{2}}{(2 h)^{2}}\right) .
\end{aligned}
$$

Because in the subtractions used for obtaining (8b), the strongly dominant principal parts canceled, expressions (8b) are less accurate than (8a). The error in the real part of $L_{\alpha_{\Delta}}$ can reach $\sim 10 \%$ and about half of that in the real part of $L_{\beta_{\Delta}}$. This affects the accuracy of (8d). The other error components remain negligible. However, all of these errors are essentially inconsequential since the simplified form of the inductances in (8b) serves only for obtaining the very simple expressions (11) for the asymptotic relative sensitivities. These results do not have to be accurate.

Later, we need expressions for the global variables $R_{i_{\Delta}}$ and $X_{i_{\Delta}}$ related to ground return. These are obtained from (8b) based on the relation $Z_{i_{\Delta}}=R_{i_{\Delta}}+j X_{i_{\Delta}}=j \omega L_{i_{\Delta}}$. Thus, for $R_{i_{\Delta}}$, we obtain

$$
\begin{aligned}
R_{\alpha_{\Delta}} & =\operatorname{Re}\left\{j \omega L_{\alpha_{\Delta}}\right\}=-\omega \operatorname{Im} L_{\alpha_{\Delta}} \\
& =\omega k H^{4} \operatorname{Im} D^{-4} \\
R_{\beta_{\Delta}} & =\operatorname{Re}\left\{j \omega L_{\beta_{\Delta}}\right\}=-\omega \operatorname{Im} L_{\beta_{\Delta}} \\
& =2 \omega k H^{2} \operatorname{Im} D^{-2} \\
R_{0_{\Delta}} & =\operatorname{Re}\left\{j \omega L_{0_{\Delta}}\right\}=-\omega \operatorname{Im} L_{0_{\Delta}} \\
& =-\omega k\left(3 \arg D+2 H^{2} \operatorname{Im} D^{-2}\right) .
\end{aligned}
$$

Similarly

$$
X_{i_{\Delta}}=\operatorname{Im}\left\{j \omega L_{i_{\Delta}}\right\}=\omega \operatorname{Re} L_{i_{\Delta}} .
$$

Equations (8c) and (8d) permit the representation of the variation with $\rho$ of the global variables $R_{i_{\Delta}}$ and $X_{i_{\Delta}}$.

\section{B. Sensitivities of $L, R$, and $X$}

We calculate the sensitivities $S_{i}=\partial L_{i} / \partial \rho$ directly from (8a) or (8b), (1a) and (1b). We note that they are proportional 
to sensitivities of the impedances $Z_{i}=j \omega L_{i}$ which include ground return but not conductor effects. They are

$$
\begin{aligned}
& S_{\alpha}=4 k \frac{H^{4}}{D^{5}} \frac{p}{\rho} \\
& S_{\beta}=4 k \frac{H^{2}}{D^{3}} \frac{p}{\rho} \\
& S_{0}=k\left(\frac{3}{D}-4 \frac{H^{2}}{D^{3}}\right) \frac{p}{\rho} .
\end{aligned}
$$

Alternatively, one might consider calculating the sensitivities by numerical differentiation of the values obtained from (7), but because some sensitivities are very small (especially for the $\alpha$-mode) the results can be inaccurate, even unusable. Equations (9) have the merit of both simplicity and high accuracy.

For practical use, we need relative sensitivities obtained from (9) with respect to either the full inductance values from (8a) or the base values from ( $8 \mathrm{~b})$. The former will be denoted by $S_{i_{r e l}}$, and the latter by $\sigma_{i}$

$$
\begin{aligned}
S_{i_{r e l}} & =S_{i} \frac{\rho}{L_{i}}=\frac{\partial L_{i}}{\partial \rho} \frac{\rho}{L_{i}}=\frac{\partial Z_{i}}{\partial \rho} \frac{\rho}{Z_{i}} \\
\sigma_{\alpha} & =S_{\alpha} \frac{\rho}{L_{\alpha_{\Delta}}}=4 k \frac{H^{4}}{D^{5}} \frac{p}{L_{\alpha_{\Delta}}} \\
\sigma_{\beta} & =S_{\beta} \frac{\rho}{L_{\beta_{\Delta}}}=4 k \frac{H^{2}}{D^{3}} \frac{p}{L_{\beta_{\Delta}}} \\
\sigma_{0} & =S_{0} \frac{\rho}{L_{0_{\Delta}}}=k\left(\frac{3}{D}-4 \frac{H^{2}}{D^{3}}\right) \frac{p}{L_{0_{\Delta}}} .
\end{aligned}
$$

For the complex sensitivities $\sigma_{i}$ in (10b), we take into account that $Z_{i_{\Delta}}=R_{i_{\Delta}}+j X_{i_{\Delta}}=j \omega L_{i_{\Delta}}$. In order to have relative sensitivities for $R_{i_{\Delta}}$ and $X_{i_{\Delta}}$, we define

$$
\sigma_{R i}=\rho \frac{\operatorname{Re} j \omega S_{i}}{R_{i_{\Delta}}}, \sigma_{X i}=\rho \frac{\operatorname{Im} j \omega S_{i}}{X_{i_{\Delta}}}
$$

where $S_{i}$ are taken from (9) and $R_{i_{\Delta}}, X_{i_{\Delta}}$ from (8c) and (8d). Thus, we obtain

$$
\begin{aligned}
\sigma_{R \alpha} & =-4 \frac{\operatorname{Im}\left\{p D^{-5}\right\}}{\operatorname{Im} D^{-4}} \\
\sigma_{R \beta} & =-2 \frac{\operatorname{Im}\left\{p D^{-3}\right\}}{\operatorname{Im} D^{-2}} \\
\sigma_{R 0} & =\frac{3 \operatorname{Im}\left\{p D^{-1}\right\}-4 H^{2} \operatorname{Im}\left\{p D^{-3}\right\}}{3 \arg D+2 H^{2} \operatorname{Im} D^{-2}}
\end{aligned}
$$

and

$$
\sigma_{X i}=\rho \frac{\operatorname{Re} S_{i}}{\operatorname{Re} L_{i_{\Delta}}} .
$$

The above sensitivities are defined in terms of real and imaginary parts of the complex components and thus they are different from those in (10b).

For the relative sensitivities (10b)-(12), we can analytically obtain limit values for both $p \rightarrow 0$ (small $\rho$ and large $\omega ; D \rightarrow$ $2 h$ ) and $p \rightarrow \infty$ (large $\rho$ and small $\omega ; D \rightarrow 2 p$ ) by using the limit values of $L_{i_{\Delta}}$ from (8b). We get

* for $p \longrightarrow 0$

$$
\sigma_{i_{0}}=\sigma_{R i_{0}}=\sigma_{X i_{0}}=0.5
$$

* for $p \rightarrow \infty$

$$
\begin{gathered}
\sigma_{i_{\infty}}=\sigma_{R 0_{\infty}}=\sigma_{X i_{\infty}}=0 \\
\sigma_{R \alpha_{\infty}}=-2.5, \sigma_{R \beta_{\infty}}=-1 .
\end{gathered}
$$

The above values indicate that the order of magnitude of all relative sensitivities could be close to 0.5 (particularly for small $\rho$ and/or large $\omega$ ) or even larger than unity. Paradoxically, it is largest [up to 2.5; see (13b)] in the case of $\alpha$-mode losses for large $p$ (large $\rho$ and/or small $\omega$ ) because the corresponding reference value $R_{\alpha_{\Delta}}$ of (8c) is very small.

Note that all inductances and relative sensitivities are functions of $\rho$ and $\omega$ only via $p$ which, as seen from (1a), depends directly on the ratio $\rho / \omega$. This simplifies the visualization of their variation, presented in Section III.

\section{Sensitivities of $H_{P}$ and $Y_{C}$}

In the calculation of transients, two main transfer functions are used: the propagation transfer function $H_{P}$ and the characteristic admittance $Y_{C}$. It is useful to know their sensitivities with respect to $\rho$ in order to choose the right accuracy for their rational approximation [4]. We have

$$
H_{P}=e^{-\sqrt{Z Y} l}, Y_{C}=\sqrt{\frac{Y}{Z}} .
$$

(The subscripts $i$ are omitted.) To obtain their relative sensitivities with respect to $\rho$, we take (10a) into account

$$
\begin{aligned}
\sigma_{H_{P}} & =\frac{\rho}{H_{P}} \frac{\partial H_{P}}{\partial \rho}=\frac{\rho}{H_{P}} \frac{\partial H_{P}}{\partial Z} \frac{\partial Z}{\partial \rho} \\
& =-\frac{\sqrt{Z Y} l}{2} \frac{\partial Z}{\partial \rho} \frac{\rho}{Z} \approx-\frac{j \omega \tau}{2} S_{r e l} \\
\sigma_{Y_{C}} & =\frac{\rho}{Y_{C}} \frac{\partial Y_{C}}{\partial \rho}=\frac{\rho}{Y_{C}} \frac{\partial Y_{C}}{\partial Z} \frac{\partial Z}{\partial \rho} \\
& =-\frac{1}{2} \frac{\partial Z}{\partial \rho} \frac{\rho}{Z}=-\frac{1}{2} S_{\text {rel }}
\end{aligned}
$$

where $\tau$ is the ideal travel time along the line.

Since $S_{\text {rel }}$ is small in the case of the line modes, (15) indicate that higher accuracy is warranted in the rational approximation of the corresponding transfer functions (14).

\section{Physical Considerations}

The reason why the losses due to ground return currents decrease as we go from the 0 -mode to the $\beta$-mode and then to the $\alpha$-mode can be explained in Fig. 1. In the upper plot, we first show the distribution (transversally to the line) of the current densities due to currents flowing in one conductor. We call 0-mode a distribution due to a single conductor current. For instance, the distribution labeled $0(+1)$ corresponds to a unit current in the conductor of phase $c$ having coordinate +1 . 

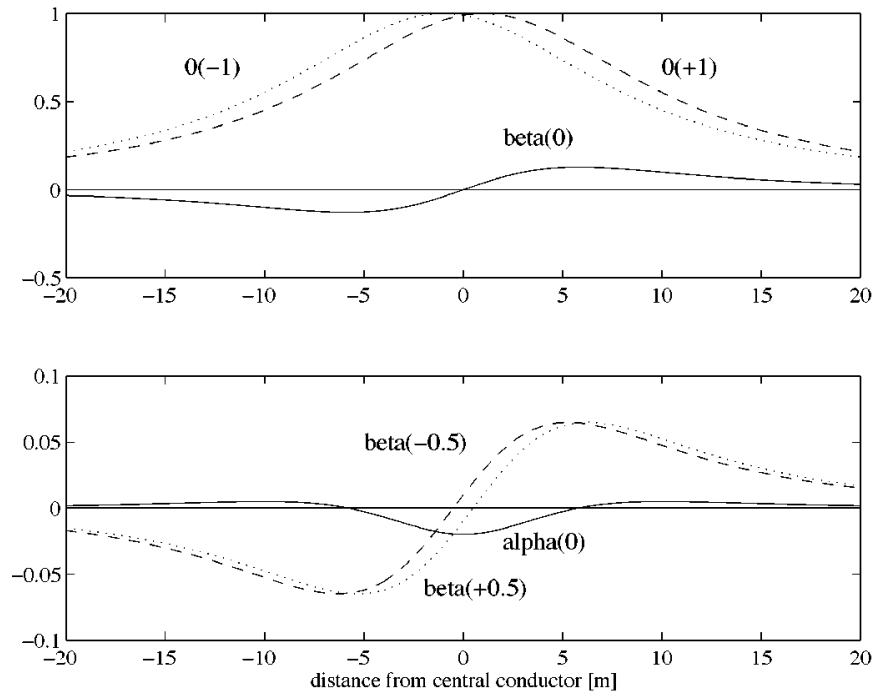

Fig. 1. Ground current distributions of $0, \beta$, and $\alpha$ modes.

The two 0 -mode distributions $0(+1)$ and $0(-1)$ (the latter for conductor $a$ ) combine as a difference for the $\beta$-mode, centered at coordinate 0 and marked as $\beta(0)$. We see that the $\beta$-mode is by an order of magnitude smaller than the 0 -modes from which it has been derived.

The lower plot of Fig. 1 shows the combination of two $\beta$-modes into an $\alpha$-mode current distribution. The plot $\beta(+0.5)$ corresponds to a $\beta$-mode centered at 0.5 , due to currents +1 in phase $c$ and -1 in phase $b$; the plot $\beta(-0.5)$ corresponds to a $\beta$-mode centered at -0.5 , due to currents -1 in phase $b$ and +1 in phase $a$. The resultant conductor currents are $\{1,-2,1\}$, clearly an $\alpha$-mode centered at 0 and labeled $\alpha(0)$. Again, due to the subtraction, the $\alpha$-mode distribution is, by an order of magnitude, weaker than the corresponding $\beta$-modes.

\section{NUMERICAL RESULTS}

In this section, we show the variation of inductances and relative sensitivities with respect to the ratio $\rho / f$, for $\rho=10^{-2}-$ $10^{4} \Omega \mathrm{m}$ and $f=10-10^{7} \mathrm{~Hz}$, for a line with horizontal configuration with height $h=8 \mathrm{~m}$, horizontal separation between phases $H=3 \mathrm{~m}$, and conductor radius $r=0.03 \mathrm{~m}$.

\section{A. Variation of $L, Z, R, X$, and of Sensitivities With $\rho / f$}

The first column of Fig. 2 shows the variation of (the magnitude of) the inductances calculated from the closed-form (8a). The inductances are shown in p.u. with respect to their value at $p$ (or $\rho / f$ ) equal to zero. Direct calculation by (3) and (7) gives almost identical results. Since for the base values to be used in the calculation of relative sensitivities, we subtract the ideal values of these inductances, i.e., their values for $p=0$, as explained for (8b), we note that, for the two line modes of Fig. 2, the inductances have a very small variation. To avoid possibly ensuing roundoff errors was the justification for the derivation of the closed-form representation (8a) of the full inductances and of the resultant differences, the base values given in (8b).

The right column of Fig. 2 shows the sensitivities of the inductances at their left (absolute values), defined in (10a). These are also sensitivities of the corresponding impedances $Z$. Note that
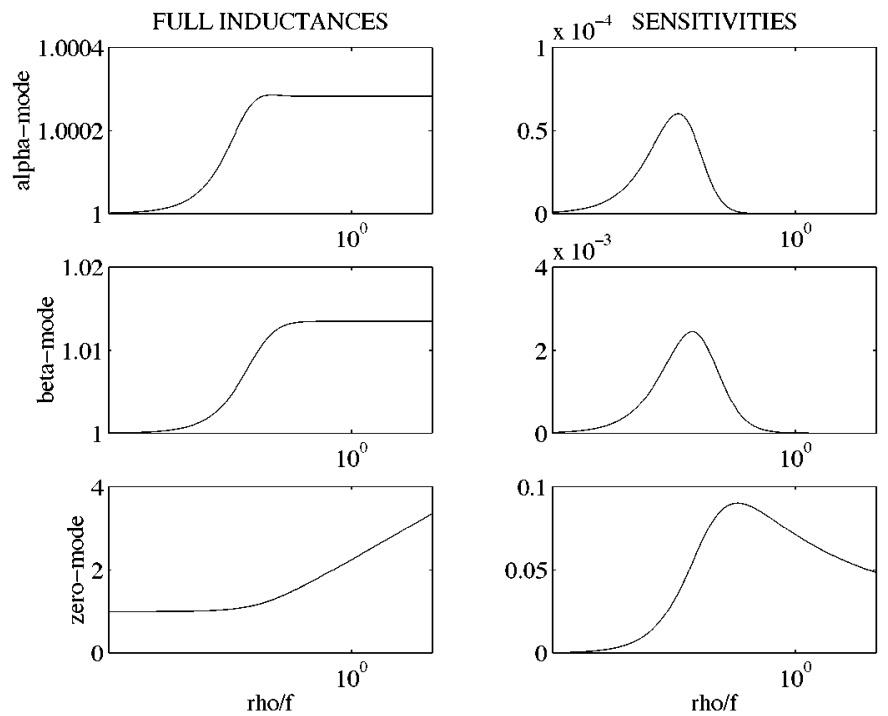

Fig. 2. Inductances from (8a) and sensitivities from (10a).
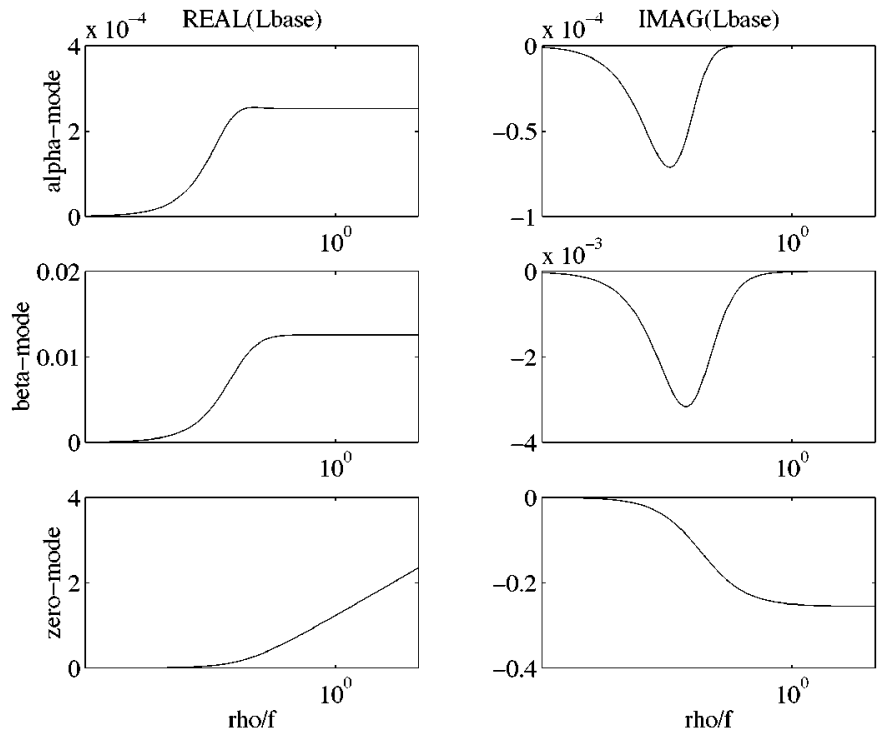

Fig. 3. Real and imaginary parts of base inductances from (8b).

the line mode sensitivities are very small-even at their peak, around $\rho / f=10^{-4}$-especially for the $\alpha$-mode. This is important in relation to the sensitivities $\sigma_{H_{P}}$ and $\sigma_{Y_{C}}$ of (15).

The base inductances are complex and Fig. 3 gives their real and imaginary parts separately. They are used directly for the calculation of the relative sensitivities. The real part is almost the same as the difference between a full inductance of Fig. 2 and its value at $p=0$. As seen from (8c) and (8d), it is the (negative of the) imaginary part that gives the line resistance $R_{i_{\Delta}}$ and the real part to which $X_{i_{\Delta}}$ is related. Thus, Fig. 3 represents also $X_{i_{\Delta}}$ (the first column) and $R_{i_{\Delta}}$ (the second column with reversed sign) in p.u., both on the basis of the corresponding full ideal ( $p=0$ ) reactance. $R_{i_{\Delta}}$ is by an order of magnitude smaller than $X_{i_{\Delta}}$, as expected. We note that the base values of the two line modes are very small, especially for the $\alpha$-mode, so that even small absolute sensitivities obtained from (9) are expected to result in significant relative values, as shown in Fig. 4. 

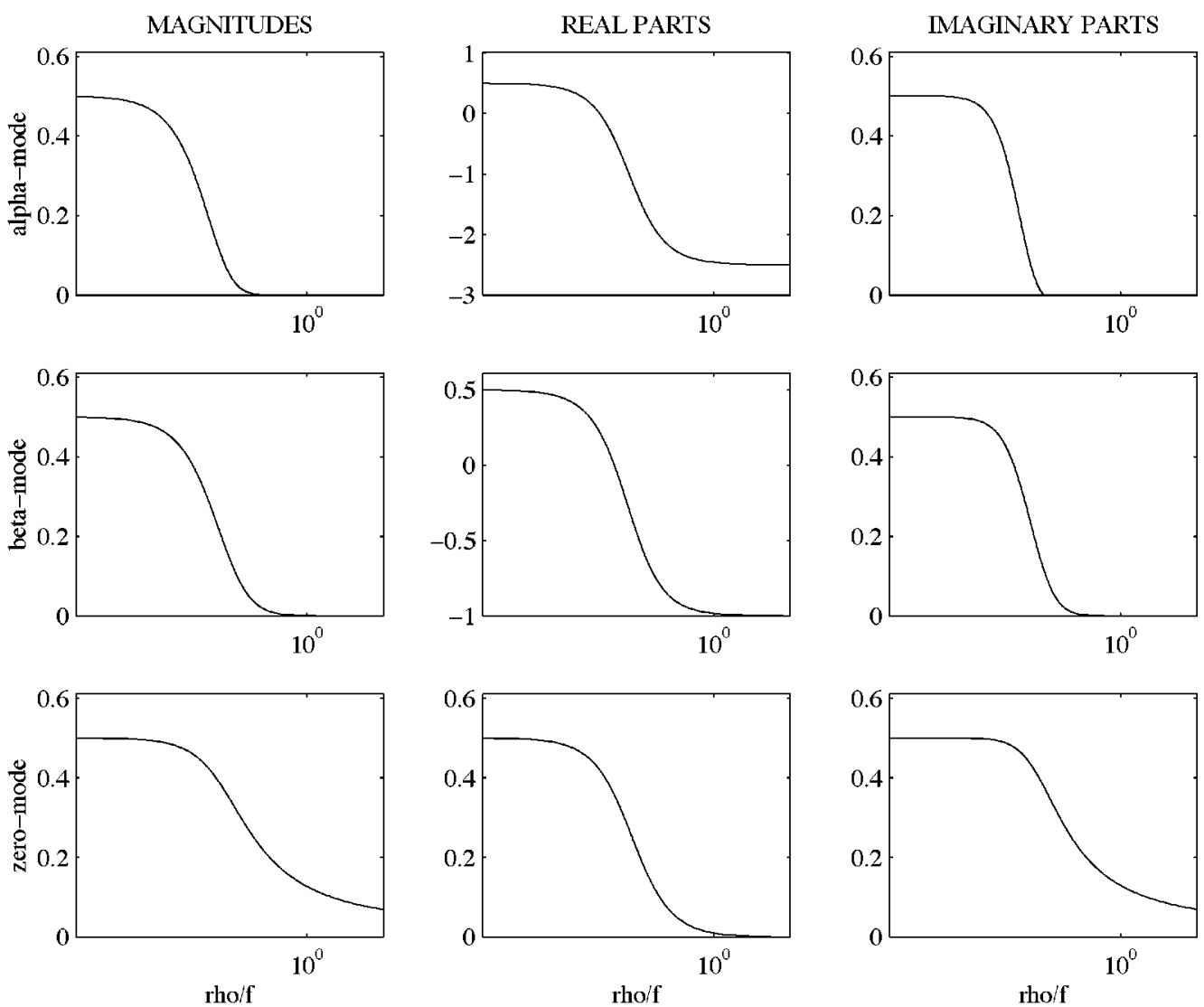

Fig. 4. Relative sensitivities. First column: magnitude, from (10b). Second column: real part, from (12a). Third column, imaginary part, from (12b).

The absolute sensitivities $S_{i},(i=\alpha, \beta, 0)$ of (9) have been defined as derivatives of $L$ with respect to $\rho$. The relative sensitivities $\sigma_{i}$ are defined separately for the magnitude, real part and imaginary part of $S_{i}$, reported to the respective base inductances, as obtained from (11). Their variation with respect to $\rho / f$ is shown in Fig. 4.

The essential observation we make is that the relative sensitivities $\sigma_{i}$ can be of the order of unity even for the two line modes, in contrast to the sensitivities $S_{r e l_{i}}$ from the second column of Fig. 2. This means that a $1 \%$ variation of $\rho$ may entail a similar variation, i.e., of roughly $1 \%$, in the relative value of both the longitudinal resistance and reactance of the transmission line. Its significance will be discussed later.

The limit values, for $p \rightarrow 0$ and $p \rightarrow \infty$, of the relative sensitivities shown in Fig. 4 are exactly those from (13a) and (13b) obtained analytically. The two nonzero values of (13b) for $p \rightarrow \infty$ are, however, of no physical significance since they correspond to vanishing sensitivities related to vanishing resistances. More meaningful are the limit values for $p \rightarrow 0$ (low resistivity, high frequency) equal to 0.5 for all relative sensitivities. Thus, quite generally, we may consider that a $1 \%$ variation of $\rho$ results in nearly $0.5 \%$ variation in the relative value of all longitudinal resistances, reactances, and impedances.

\section{B. Particular Case: $\rho=100 \Omega m, f=100 \mathrm{KHz}$}

The particular case below is presented to show some numbers, real or complex, in order to clarify the meaning of the results obtained. For $\rho=100 \Omega \mathrm{m}, f=100 \mathrm{kHz}$, we have $\rho / f=10^{-3}$ and we get the following results. Full inductances $[\mathrm{H} / \mathrm{m}]$

$$
\begin{aligned}
& L_{\alpha}=8.7483 e-007-9.5380 e-012 j \\
& L_{\beta}=1.0580 e-006-2.2225 e-009 j \\
& L_{0}=2.3156 e-006-2.7532 e-007 j .
\end{aligned}
$$

Their accuracy (relative value) is better than $10^{-5}$. The corresponding impedances, $Z_{i}=j \omega L_{i}[\Omega / \mathrm{m}]$, are

$$
\begin{aligned}
& Z_{\alpha}=5.9929 e-006+5.4967 e-001 j \\
& Z_{\beta}=1.3964 e-003+6.6473 e-001 j \\
& Z_{0}=1.7299 e-001+1.4550 e+000 j .
\end{aligned}
$$

We note that the resistive part of the line modes became very small, especially for the $\alpha$-mode, while the reactance portion is not very different. This is of course due to the inductance in air, above ground.

Next we show the base inductances, $[\mathrm{H} / \mathrm{m}]$, of $(8 \mathrm{~b})$

$$
\begin{aligned}
& L_{\alpha_{\Delta}}=2.4996 e-010-9.6252 e-012 j \\
& L_{\beta_{\Delta}}=1.2359 e-008-2.2607 e-009 j \\
& L_{0_{\Delta}}=4.6857 e-007-2.7529 e-007 j .
\end{aligned}
$$

For these we obtain the impedances $Z_{i_{\Delta}}=j \omega L_{i_{\Delta}}[\Omega / \mathrm{m}]$

$$
\begin{aligned}
Z_{\alpha_{\Delta}} & =6.0477 e-006+1.5705 e-004 j \\
Z_{\beta_{\Delta}} & =1.4205 e-003+7.7657 e-003 j \\
Z_{0_{\Delta}} & =1.7297 e-001+2.9441 e-001 j .
\end{aligned}
$$


If we compare the initial, full inductances and impedances of (16) and (17) with the new values of (18) and (19), respectively, we see that the subtraction process has mainly affected the real part of the inductances and the imaginary part of the impedances. Still, the line mode resistances in (19) are by an order of magnitude smaller than the corresponding reactances (consistent with the base inductance values in Fig. 3).

The significance of the small resistance values for the line modes can be explained in terms of voltage drops and losses on the transmission line, as follows.

In a transient the voltage drops and the resultant voltages will be determined by the impedance of the line and will thus be primarily affected by its reactance. The resistance will affect only the losses and thereby the attenuation of the natural component of the transient. This attenuation is very small in the two line modes of (17) and (19), but if the objective of the study is to focus on the attenuation, then what matters is the real part of the impedances and that should be taken as base value. In the resultant transient, attenuation in a line mode is small and may have a secondary significance.

It is also important to note that the resistances we discussed above do not include the resistances of the conductors. If the latter are to be taken into account, then they have to be added to the base values defined for earth return effects alone. The result is that the resultant sensitivities for the line modes will be smaller than the values shown in Fig. 4.

The fact that transients are determined by the full impedances rather than by the difference or base values, is reflected in (15) for the sensitivities of transfer functions $H_{P}$ and $Y_{C}$. These are directly proportional to $S_{r e l}$. In our case

$$
\begin{aligned}
S_{r e l_{\alpha}} & =1.4449 e-005 \\
S_{r e l_{\beta}} & =1.6884 e-003 \\
S_{r e l_{0}} & =8.0733 e-002 .
\end{aligned}
$$

If the line length is $l=300 \mathrm{~km}$, then $\tau=1 \mathrm{~ms}$ and in (15a) $\omega \tau=300$. Thus, we get the relative sensitivities

$$
\begin{aligned}
\left|\sigma_{H_{P \alpha}}\right| & =2.1674 e-003\left|\sigma_{Y_{C \alpha}}\right|=7.2245 e-004 \\
\left|\sigma_{H_{P \beta}}\right| & =2.5326 e-001\left|\sigma_{Y_{C \beta}}\right|=0.8842 e-003 \\
\left|\sigma_{H_{P 0}}\right| & =12.1099 \quad\left|\sigma_{Y_{C 0}}\right|=4.0366 e-002 .
\end{aligned}
$$

Note that the line mode sensitivities are smaller than for the ground. Because $\sigma_{H_{p}}$ of (15a) is proportional to frequency, its value in (21) for the ground mode became inordinately large for $f=100 \mathrm{kHz}$. At this frequency, $H_{P}$ of (14) itself is extremely small for the ground mode, so this fact is of no significance. This will be discussed in more detail in the next section.

\section{Sensitivities of $H_{P}$ and $Y_{C}$}

By (15b), the sensitivity of $Y_{C}$ is half of the value of $S_{\text {rel }}$ shown in the right column of Fig. 2. However, by (15a), the sensitivity of $H_{P}$ depends also directly on frequency and the length of the line. Therefore, in Fig. 5, we focus on a single resistivity, $\rho=100 \Omega \mathrm{m}$ and represent $\left|\sigma_{H_{P}}\right|$ as a function of $f$.

The first column shows the variation of $\left|\sigma_{H_{P}}\right|$, the second column shows $\left|H_{P}\right|$ itself, for two line lengths $50 \mathrm{~km}$ and $500 \mathrm{~km}$. Also shown for $\left|H_{P}\right|$ are the two limits corresponding to $\pm 40 \%$ variations in $\rho$, based on the corresponding relative
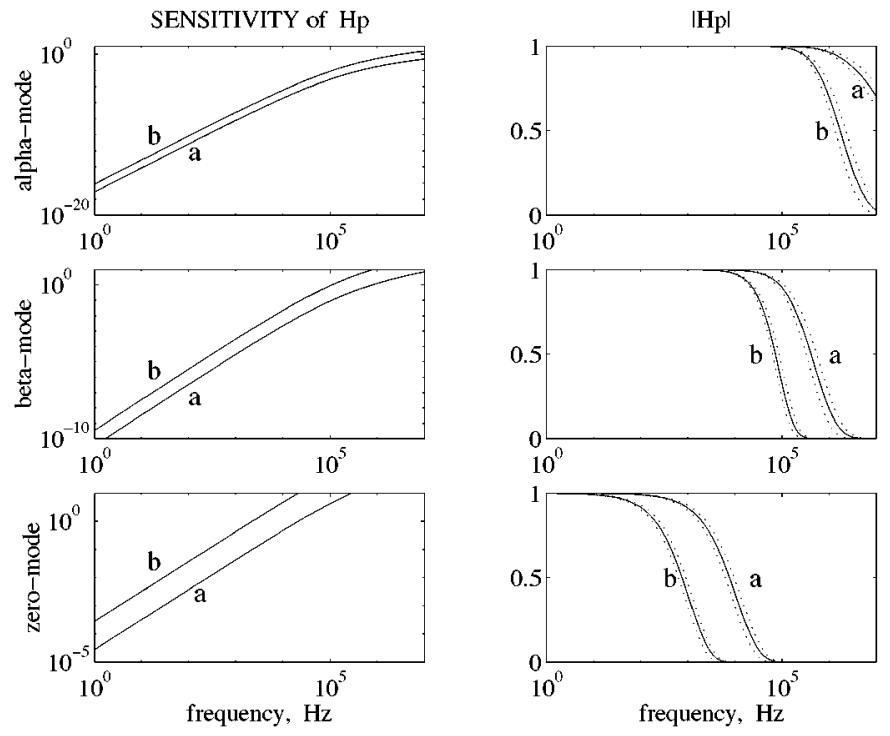

Fig. 5. Sensitivity $\left|\sigma_{H_{P}}\right|$ and propagation function $\left|H_{P}\right|$ for $\rho=100 \Omega \mathrm{m}$. Line lengths: $a=50 \mathrm{~km}, b=500 \mathrm{~km}$.

sensitivities $\left|\sigma_{H_{P}}\right|$ (checked against limits calculated directly by varying $\rho$ ).

We see that the sensitivities increase with frequency and line lengths and we note the following.

1) For the line modes $\left|\sigma_{H_{P}}\right|$ is smaller than for the ground mode.

2) In all cases, the sensitivities are very small for low frequencies defined by the initial, flat portion of $\left|H_{P}\right|$ and thus this portion should be very accurately reproduced in the fitting process. Its frequency range is highest for the two line modes.

3) Conversely, the sensitivities become large at the very high frequencies where $\left|H_{P}\right|$ vanishes, but there the idea of relative accuracy is irrelevant.

4) At the downward slope of $\left|H_{P}\right|$, the relative sensitivities increase progressively so that here accurate fitting becomes less important (not just because $\left|H_{P}\right|$ is small). Thus, for all modes, accurate fitting is unnecessary at the respective higher frequencies which however are much lower for the ground mode than for the two line modes.

\section{Simulation Results}

A small, 5-line test system has been used for assessing the effect of varying the earth resistivity on the simulated transients. A unit step voltage has been applied and the resulting current has been calculated for $\rho=100 \Omega \mathrm{m}$ and then for $\rho=200 \Omega \mathrm{m}$, using high-accuracy fitting and simulations.

For a pure ground mode input, the output varied by $7.5 \%$, while for a pure line mode it varied by only $0.5 \%$. Clearly, the effect of ground resistivity on transients is significant for a ground mode but minimal for a line mode (not including aspects of damping).

These time domain simulation outputs are related to the frequency domain results of this paper only by a (Fourier-type) transformation from frequency to time domain and provide therefore only an approximate, qualitative confirmation of the latter. They are biased toward lower frequencies due to the step input. 


\section{CONCLUSIONS}

In an attempt to examine the effect of the generally poorly known ground resistivity on transmission line transients, this paper focused on its effect on the variation of the longitudinal transmission line parameters: inductances, impedances, reactances, and resistances.

It has been found that the corresponding relative sensitivities are close to 0.5 . This means that a $1 \%$ variation in $\rho$ may result in a nearly $0.5 \%$ change in some features of a transient, such as its attenuation. Surprisingly, this characterization applies also for line modes, even for the $\alpha$-component in a horizontal line configuration. At the same time, however, the sensitivity of the line mode transfer functions used in the calculation of transients is generally very small, especially for the $\alpha$-component, indicating that the line mode computations can and should be carried out with high precision so that even damping effects are reliably calculated. The effect of the conductor resistance, not taken into account in the analysis, will strongly reduce the significance of uncertainties in the small line-mode ground return effects.

In practical applications, these results have to be interpreted and used cautiously. However, they suggest quite generally that very high accuracy in computer simulations may be excessive and should be avoided whenever fast simulation is important. The use of lower order rational approximation for the ground mode may improve the efficiency of simulation by significantly reducing the computation time.

\section{ACKNOWLEDGMENT}

The author gratefully acknowledges the contribution of W. do Couto Boaventura, who performed the simulations in Section III-D.

\section{REFERENCES}

[1] A. Semlyen and A. Dabuleanu, "Fast and accurate switching transient calculations on transmission lines with ground return using recursive convolutions," IEEE Trans. Power App. Syst., vol. PAS-94, pp. 561-571, Mar./Apr. 1975.

[2] J. R. Marti, "Accurate modeling of frequency-dependent transmission lines in electromagnetic transient simulations," IEEE Trans. Power App. Syst., vol. PAS-101, pp. 147-157, Jan. 1982.

[3] A. Morched, B. Gustavsen, and M. Tartibi, "A universal model for accurate calculation of electromagnetic transients on overhead lines and underground cables," IEEE Trans. Power Delivery, vol. 14, pp. 1032-1038, July 1999.

[4] B. Gustavsen and A. Semlyen, "Rational approximation of frequency domain responses by vector fitting," IEEE Trans. Power Delivery, vol. 14, pp. 1052-1061, July 1999.

[5] A. O. Soysal and A. Semlyen, "Reduced order transmission line modeling for improved efficiency in the calculation of electromagnetic transients," IEEE Trans. Power Syst., vol. 9, pp. 1494-1498, Aug. 1994.

[6] A. Deri, G. Tevan, A. Semlyen, and A. Castanheira, "The complex ground return plane: A simplified model for homogeneous and multilayer earth return," IEEE Trans. Power App. Syst., vol. PAS-100, pp. 3686-3693, Aug. 1981.

[7] X. Wang, D. A. Woodford, R. Kuffel, and R. Wierckx, "A real-time transmission line model for a digital TNA," IEEE Trans. Power Delivery, vol. 11, pp. 1092-1097, Apr. 1996.

[8] A. S. Morched, J. H. Ottevangers, and L. Marti, "Multi-Port frequency dependent network equivalents for the EMTP," IEEE Trans. Power Delivery, vol. 8, pp. 1402-1412, July 1993.

Adam Semlyen (SM'70-F'88-LF'97) was born in Gherla, Rumania on January 10, 1923. He received the Dipl.Ing. and Ph.D. degrees from the Polytechnic Institute of Timisoara, Rumania, in 1949 and 1965, respectively.

He held academic positions at the Polytechnic Institute of Timisoara. In 1969, he joined the University of Toronto, Toronto, ON, Canada, where he has been a Professor in the Department of Electrical and Computer Engineering, since 1988. His research interests include steady-state and dynamic analysis, as well as the computation of electromagnetic transients in power systems.

Dr. Semlyen received the Fellow Award for contributions to the theory and analysis of electromagnetic transients and harmonics in electric power systems. 